\title{
Syzygium aromaticum L. (Clove) Essential Oil as a Reducing Agent for the Green Synthesis of Silver Nanoparticles
}

\author{
Matheus Vinicius de Oliveira Brisola Maciel1*, Aline da Rosa Almeida1, \\ Michelle Heck Machado', Ana Paula Zapelini de Melo', \\ Cleonice Gonçalves da Rosa ${ }^{1}$, Daniele Ziglia de Freitas ${ }^{1}$, \\ Carolina Montanheiro Noronha ${ }^{1}$, Gerson Lopes Teixeira ${ }^{1}$, \\ Rafael Dutra de Armas ${ }^{2}$, Pedro Luiz Manique Barreto ${ }^{1}$ \\ ${ }^{1}$ Departamento de Ciência e Tecnologia de Alimentos, Universidade Federal de Santa Catarina, Florianópolis, Brasil \\ ${ }^{2}$ Centro Universitário-Católica de Santa Catarina, Joinville, Brasil \\ Email: *matheusmaciel1@hotmail.com
}

How to cite this paper: Maciel, M.V.O.B., Almeida, A.R., Machado, M.H., de Melo, A.P.Z., da Rosa, C.G., de Freitas, D.Z., Noronha, C.M., Teixeira, G.L., de Armas, R.D. and Barreto, P.L.M. (2019) Syzygium aromaticum L. (Clove) Essential Oil as a Reducing Agent for the Green Synthesis of Silver Nanoparticles. Open Journal of Applied Sciences, 9, 45-54.

https://doi.org/10.4236/ojapps.2019.92005

Received: January 7, 2019

Accepted: January 28, 2019

Published: January 31, 2019

Copyright $\odot 2019$ by author(s) and Scientific Research Publishing Inc. This work is licensed under the Creative Commons Attribution International License (CC BY 4.0).

http://creativecommons.org/licenses/by/4.0/

\begin{abstract}
The present investigation details a green synthesis of silver nanoparticles (AgNP) using the essential oil of Syzygium aromaticum L. (clove) as reducing agent, which is a matrix with a high content of eugenol, an important compound for the reducing action of silver nitrate. The synthesis of AgNP was performed at different $\mathrm{pH}$ conditions ( $\mathrm{pH} \mathrm{7,8,9}$ and 10), and was monitored by UV-Vis Spectroscopy, Dynamic Light Scattering (DLS) and Transmission Electron Microscopy. The synthesized nanoparticles presented characteristic Surface Plasmon Resonance bands with maximum absorbance between 405 and $460 \mathrm{~nm}$. The DLS analysis revealed particle sizes from 31 to $72 \mathrm{~nm}$ and zeta potential between -30.1 and $-50.8 \mathrm{mV}$ indicating good stability against the agglomeration of the particles in solution. The micrographs obtained by TEM showed different particle shapes and a predominance of spherical-shaped nanoparticles, and average size ranging from 27 to $94 \mathrm{~nm}$. The clove-based silver nanoparticles were efficient in controlling the growth of Escherichia coli and Staphylococcus aureus bacteria, and the minimum inhibitory concentration ranged from 60 to $100 \mu \mathrm{L} / \mathrm{mL}$. This study highlights the feasibility of clove essential oil as an alternative for the synthesis of silver nanoparticles by a simple, inexpensive and eco-friendly method.
\end{abstract}

\section{Keywords}

Essential Oil, Clove, Eugenol, Silver Nanoparticles, Antibacterial Activity 


\section{Introduction}

A vast number of physical, chemical and biological methods can be applied to obtain metallic nanoparticles. Biological methods are those employing microorganisms or plants for the nanoparticle biosynthesis [1] [2] [3]. The use of plants or plant derivatives for the synthesis of nanoparticles is widely used nowadays, and their compounds often have important biological functionalities that can act synergistically with the nanoparticles for various applications in different areas from the food to the pharmaceutical and medical industries. The concern involving the non-degradation of the environment is even more evident today, which highlights the importance of using sustainable industrial methods capable of proposing alternatives for the reduction of harmful chemical residues [4] [5] [6].

In this context, silver nanoparticles (AgNP) are prominent in applications as antimicrobial agents that can eliminate microorganisms by interacting with their cells, thus preventing the cellular respiration, and consequently its replication [7]. Whereas, they are non-toxic against eukaryotic cells, especially at the concentrations usually used. However, the AgNP synthesis using harmful chemicals may limit their application in some areas. Therefore, the search for alternative agents capable of reducing silver salts for the formation of nanoparticles, without causing health or environmental risks, is an important role in science [8].

The clove (Syzygium aromaticum L.) is a plant grown in several countries including Brazil. The clove essential oil is widely used for its antimicrobial, antifungal and antioxidant action, as well as anesthetic and analgesic effects. These characteristics are strongly attributed to its major compound, eugenol, present in the oil in concentrations that can reach up to 90\% [5] [9]. The chemical composition suggests clove essential oil as a potential silver reducing agent for the synthesis of nanoparticles. The probable mechanism of reduction is given by the proton donation of the eugenol structure, reducing the $\mathrm{Ag}^{+}$to $\mathrm{Ag}^{0}$ and thus forming the nanoparticles. In addition, the phytochemicals present in the essential oil can also interact on the surface of the nanoparticles, resulting in stabilization and preservation [10] [11].

The use of plant extracts in the synthesis of silver nanoparticles is quite common. However, there is still a shortage in the use of essential oils for this purpose. Therefore, the main objective of the present study was to evaluate a green synthesis of silver nanoparticles using the essential oil of Syzygium aromaticum L. (clove) as a reducing and stabilizing agent, as well as their characterization by transmission electron microscopy, dynamic light scattering, UV-Vis spectroscopy, besides the evaluation of the antimicrobial activity.

\section{Materials and Methods}

\subsection{Materials}

Silver nitrate $\left(\mathrm{AgNO}_{3}\right)$ and acetone were obtained from Sigma Aldrich (Brazil). Clove (Syzygium aromaticum L.) essential oils were purchased from the local 
market of Florianópolis (Santa Catarina, Brazil). All chemicals used were of analytical grade, and the solutions were prepared with deionized water.

\subsection{Green Synthesis of AgNPs Using Clove Essential Oil}

Initially, the clove essential oil was diluted in acetone at a ratio of 1:170. A stock solution of $0.31 \mathrm{mmol} . \mathrm{L}^{-1}$ silver nitrate $\left(\mathrm{AgNO}_{3}\right)$ was prepared. Four aliquots of this solution were collected, and their $\mathrm{pH}$ was adjusted to 7, 8, 9 and 10 with a 0.1 mol. $\mathrm{L}^{-1} \mathrm{NaOH}$ solution in order to evaluate the synthesis under the different conditions. A 30-mL aliquot of each solution was heated under constant magnetic stirring, and $2 \mathrm{~mL}$ of the diluted clove essential oil was added dropwise into the boiling solution. Afterward, the solution remained under heating and vigorous stirring for $30 \mathrm{~min}$. A brown-yellow color was observed indicating the formation of silver nanoparticles (AgNP).

\subsection{UV-Vis Spectroscopy}

The UV-Vis spectroscopy absorption study was performed on a Hitachi spectrophotometer, model U-1800 (Tokyo, Japan) at the wavelength range of 300 to $800 \mathrm{~nm}$. The readings were performed at $\sim 25^{\circ} \mathrm{C}$, and the results were used to build the curves of the Surface Plasmon Resonance (SPR) bands of the samples.

\subsection{Dynamic Light Scattering (DLS) Analysis}

For the dynamic light scattering analysis, the AgNPs samples were evaluated in a Zetasizer Nano Series (Malvern Instruments Worcestershire, UK) at a fixed angle of $173^{\circ}$ at room temperature $\left(25^{\circ} \mathrm{C}\right)$. The results of particle size (Z-ave), polydispersity index $(\mathrm{PI})$ and the zeta potential $(\zeta)$ were obtained.

\subsection{Transmission Electron Microscopy (TEM)}

Transmission electron microscopy was performed to elucidate the morphology of AgNPs in the different synthesis conditions. A JEOL model JEM-1011 (Tokyo, Japan) microscope was used for the TEM evaluation under $80 \mathrm{kV}$. The obtained images and the size distribution of the particles were analyzed with the aid of the Quantikov Image Analyzer software.

\subsection{Antimicrobial Activity of AgNPs}

The evaluation of the antimicrobial activity of AgNPs was performed according to a methodology adapted from the Clinical and Laboratory Standards Institute (CLSI) [12]. The AgNP samples were diluted with ultrapure water to obtain solutions at final concentrations in the wells of $40,60,80$ and $100 \mu \mathrm{L} \cdot \mathrm{mL}^{-1}$. The following bacterial cultures were used: Escherichia coli (ATCC 8739) (gram negative), Staphylococcus aureus (ATCC 25923) (gram positive) and Bacillus cereus (ATCC 10987) (gram positive). Bacterial cells were suspended in $0.9 \%(\mathrm{w} / \mathrm{v}) \mathrm{sa}-$ line solution $(\mathrm{NaCl})$ and the inoculum density was standardized using the McFarland turbidity comparison standard to achieve a final plate concentration 
of $\sim 10^{5} \mathrm{CFU} / \mathrm{mL}$.

Sterile 96-well polypropylene microplates were used for analysis. In each well were added $5 \mu \mathrm{l}$ of inoculum, $90 \mu \mathrm{L}$ of Mueller-Hinton broth and $10 \mu \mathrm{L}$ of nanoparticles at different concentrations. Plates were incubated at $35^{\circ} \mathrm{C}$ for $16-20$ h. Afterward, the turbidity was observed (to the naked eye) and compared to the negative control (without inoculum) and to the positive control (without nanoparticles), being considered efficient in controlling the microorganism when the turbidity was not observed.

\subsection{Statistical Analysis}

All assays were performed at least in triplicate, and the data were expressed as mean and standard deviation.

\section{Results and Discussion}

To verify the efficiency of AgNP synthesis at different pHs using clove essential oil as reducing agent, the samples were analyzed by UV-Vis spectroscopy from 300 to $800 \mathrm{~nm}$ and the particle's formation was observed by the Surface Plasmon Resonance bands.

The formation of AgNPs occurred due to the reduction of $\mathrm{Ag}^{+}$ions into $\mathrm{Ag}$ atoms by the clove essential oil added to the $\mathrm{AgNO}_{3}$ solution, which was confirmed after the colorless solution was turned into a yellowish brown. The maximum formation of AgNPs occurred at $\mathrm{pH} 8$ (max absorbance at $435 \mathrm{~nm}$ ), followed by pH 9, 10 and 7 (Figure 1). The clove essential oil has 70\%-90\% of the eugenol compound in its chemical composition, in addition to other compounds in smaller amounts. These compounds present a variety of functional groups with high affinity for noble metals aiding in the stabilization of the nanoparticles [10]. Additionally, the presence of a phenolic ring in the eugenol structure allows it to act as a reducing agent by donating protons $\left(\mathrm{H}^{+}\right)$. Thus, it is

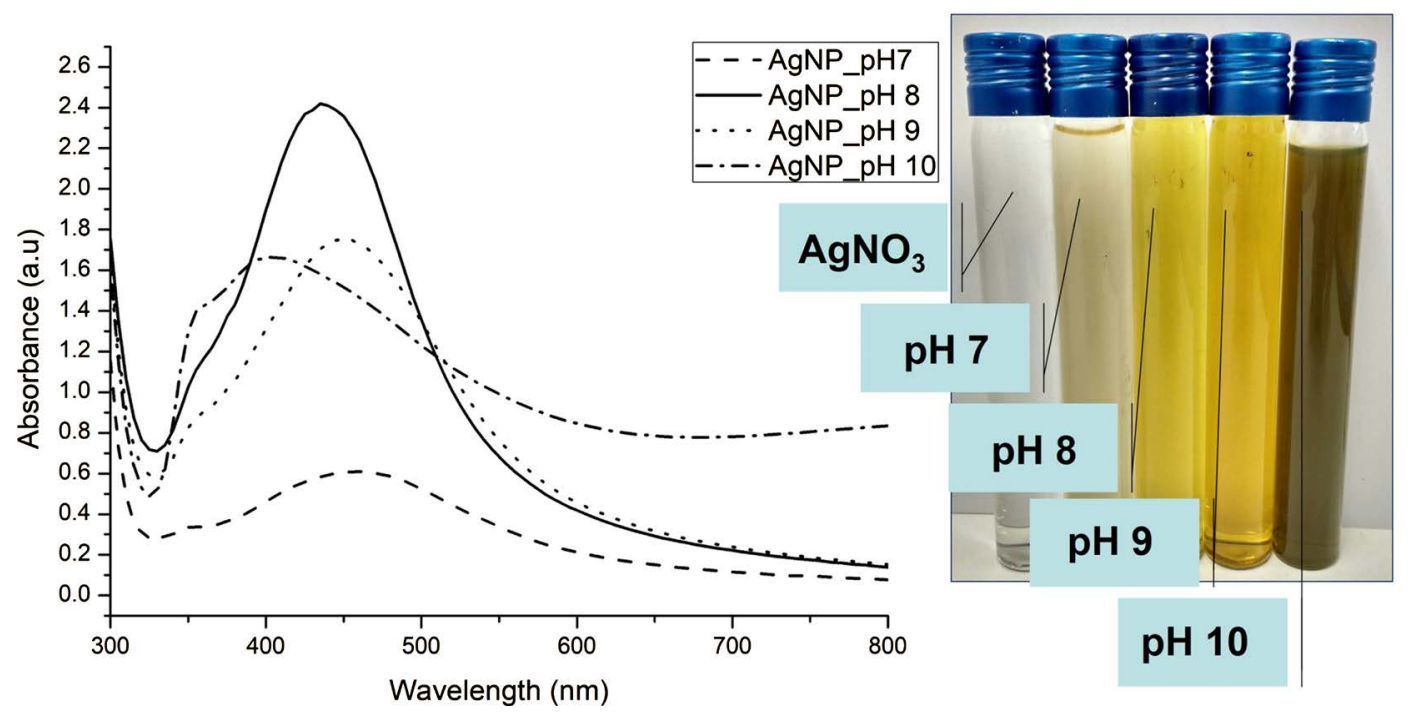

Figure 1. The SPR band for AgNPs using different $\mathrm{pH}$ condition and matching color. 
suggested that the reduction of silver nitrate by clove essential oil occurs mainly by the action of the eugenol, forming the nanoparticles [13].

Bands of SPR with maximum absorbance at $430 \mathrm{~nm}$ are reported for the synthesis of nanoparticles using an aqueous clove extract [10], while a maximum absorbance of $435 \mathrm{~nm}$ is [14] reported for Cinnamon zeylanicum silver nanoparticles, which are similar to those found in the present work.

From Figure 1 is possible to observe the difference in the color of the samples compared to the $\mathrm{AgNO}_{3}$ solution which indicates the formation of the nanoparticles. This was corroborated by the SPR absorption bands that confirmed the formation of AgNPs in all $\mathrm{pH}$ conditions. In addition to the higher peak absorbance intensity for the sample synthesized at $\mathrm{pH} 8$, its SPR band is more well-defined and indicates a better formation of nanoparticles, consistent with other results found in the literature [4] [6]. The full width at half-height (FWHH) indicates the particle size distribution, i.e., wider bands are representative of high dispersion index and diverse formats [3], which can be observed in the band of AgNP synthesized at pH 7 and 10.

\subsection{Size Distribution and Characterization}

The samples were characterized for the elucidation of their size, polydispersity index and zeta potential by DLS, while the morphology of silver nanoparticles was studied by transmission electron microscopy (TEM) to verify their shape. The results of the DLS measurements are shown in Table 1.

The mean size obtained by DLS indicates the hydrodynamic radius of the particle. A higher deviation is verified for the sample AgNP_pH 7, confirming what was observed in the SPR band. The AgNP_pH 10 sample showed the smallest average particle size; however, the highest polydispersity index (PI) was also observed for this sample. In this case, PI values lower than 0.4 indicate populations with a narrower distribution [15]. This result reinforces the results observed in the UV-Vis analysis. All the AgNP samples showed zeta potential values between -30.1 and $-50.8 \mathrm{mV}$ indicating good stability against agglomeration.

Figure 2 shows the micrographs of AgNP obtained by TEM. Dense particles of various shapes and a thin layer on the surface of silver nanoparticles were observed. This may be evidence of a binding between the oil's phytochemicals, responsible for promoting steric hindrance stability. The different particle shapes observed herein can be attributed to the $\mathrm{pH}$ of the solutions that influences the

Table 1. Results of the dynamic light scattering analysis (DLS) for the AgNPs samples at different $\mathrm{pHs}$ represented by mean values and standard deviation.

\begin{tabular}{cccc}
\hline Sample & Average Size $(\mathrm{nm})$ & Polydispersity Index & Zeta $(\mathrm{mV})$ \\
\hline AgNP_pH 7 & $71.9 \pm 14.5$ & $0.269 \pm 0.043$ & $-31.9 \pm 8.5$ \\
AgNP_pH 8 & $55.4 \pm 4.1$ & $0.383 \pm 0.019$ & $-34.8 \pm 3.6$ \\
AgNP_pH 9 & $74.8 \pm 2.5$ & $0.232 \pm 0.011$ & $-30.1 \pm 0.7$ \\
AgNP_pH 10 & $31.4 \pm 5.8$ & $0.543 \pm 0.004$ & $-50.8 \pm 0.8$ \\
\hline
\end{tabular}




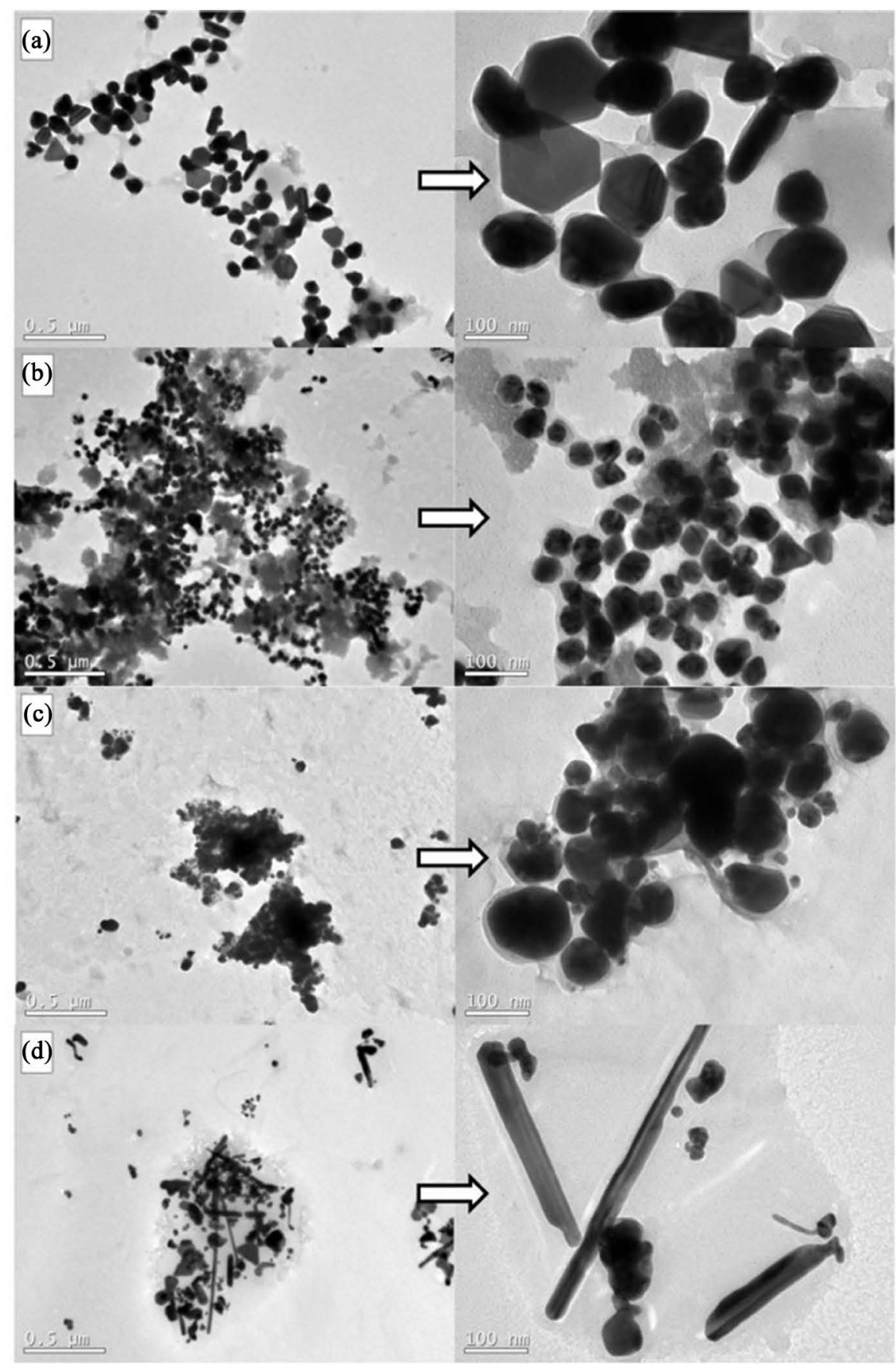

Figure 2. Transmission electron microscopy (TEM) for AgNPs synthesized with clove essential oil at different $\mathrm{pH}$ conditions. (a) $\mathrm{pH}$ 7; (b) $\mathrm{pH}$ 8; (c) $\mathrm{pH}$ 9; (d) $\mathrm{pH} 10$.

reduction and stabilization of the nanoparticles system [16] [17].

The sample AgNP_pH 7 (Figure 2(a)) showed mainly triangles, squares, hexagons, and spheres, while pentagons, hexagons, and spherical shapes were observed for AgNP_pH 8 (Figure 2(b)), and little number of triangular shapes. In AgNP_pH 9 (Figure 2(c)) the spherical shapes were predominant, but a larger agglomeration of the particles was observed, which in this case, can contribute to the coalescence of the particles. The largest variety of shapes was observed in 
AgNP_pH 10 (Figure 2(d)) which presented hexagon, square, spherical and needle formats.

The micrographs were analyzed to obtain the size distribution with the aid of the Quantikov Image Analyzer software, and the histograms of size distribution are represented in Figure 3. Overall, the samples presented a mean particle diameter between 27 to $94 \mathrm{~nm}$. The AgNP_pH 7 sample had the highest average diameter and a substantial heterogeneity of sizes $(78.0 \pm 16.3 \mathrm{~nm})$, this result reinforces what was observed by UV-Vis (Figure 1) and resembles the results obtained by DLS (Table 1). The lowest mean diameter was observed for the sample AgNP_pH $8(35.5 \pm 8.5 \mathrm{~nm})$, which showed $76 \%$ of the particles with a diameter in the $40 \mathrm{~nm}$ range, showing a high homogeneity in its particle sizes. The AgNP_pH 9 sample had a mean diameter of $44.0 \pm 18.9 \mathrm{~nm}$ indicating a high degree of heterogeneity like AgNP_pH 7, which can be evidenced in TEM micrographs (Figure 2). Furthermore, the average diameter of the AgNP_pH 10 sample (37.9 $\pm 18.7 \mathrm{~nm})$ was quite similar to that obtained by DLS (Table 1), and its high standard deviation indicates the significant dispersion of the particles, with a great variety of shapes, which reinforces the results of SPR and TEM.
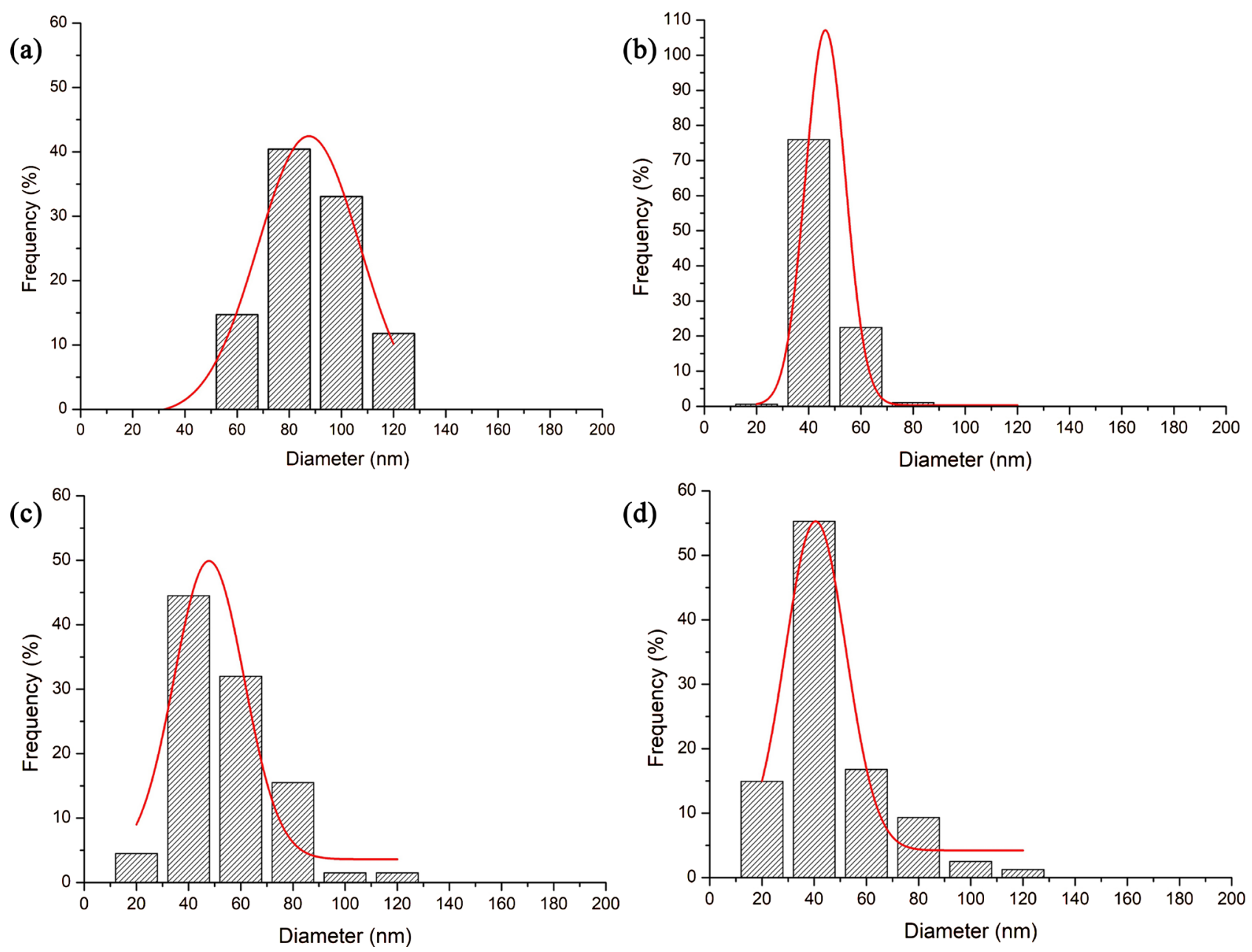

Figure 3. Size distribution histograms of AgNPs synthesized at different $\mathrm{pH}$ using clove essential oil. 


\subsection{Evaluation of Antimicrobial Activity}

The broth microdilution test was used to determine the minimum inhibitory concentration (MIC) of the AgNPs. Turbidity was the parameter used to verify the growth of the microorganism in the wells. The sample AgNP pH 7 presented MIC of $60 \mu \mathrm{L} / \mathrm{mL}$ against E. coli, which is the same found for AgNP pH 8. For $S$. aureus the MIC of AgNP pH 8 was $80 \mu \mathrm{L} / \mathrm{mL}$. The AgNP pH 9 sample presented a MIC of $100 \mu \mathrm{L} / \mathrm{mL}$ for both microorganisms. At $\mathrm{pH} 10$ no inhibition of bacterial growth was observed. This result can be attributed to the higher variation on the particles' shapes and lower volume of formation in this condition. Additionally, inhibition did not occur in any of the samples tested against B. cereus. Reference [18] reports that triangular shaped particles exhibit almost total inhibition of bacterial growth, which decreases for spherical-shaped particles and is even smaller for the stem-shaped particles. It is also reported antimicrobial activity of silver nanoparticles against $S$. aureus and $E$. coli by the agar diffusion method [4]. Similar results are demonstrated by [13] against the same bacteria using polymer films impregnated with silver nanoparticles. The MIC of $8 \mu \mathrm{g} / \mathrm{mL}$ is reported for nanoparticles measuring $\sim 20 \mathrm{~nm}$ against $E$. coli [7]. The results observed in this study are consistent with those found in the literature, regarding the particularities of particle size and shape.

\section{Conclusion}

The present work demonstrates a green synthesis of silver nanoparticles using the essential oil of clove which is a matrix rich in eugenol compound, essential for the action of silver nitrate reduction and synthesis of the nanoparticles. Particles of different shapes and predominance of spherical shapes were obtained by a simple, low cost and eco-friendly method. The antimicrobial activity of AgNPs was tested and proven to be effective against bacteria such as E. coli and $S$. aureus at $\mathrm{pH} 7,8$ and 9, being more effective at $\mathrm{pH} 8$, reinforcing the effect of the shape associated with volume and size. These results were achieved using low concentrations of the sample, showing the advantage of using clove essential oil with a high concentration of the active compound.

\section{Acknowledgements}

The authors would like to thank the LCME-UFSC for technical support during electron microscopy work. This study was financed in part by the Coordenação de Aperfeiçoamento de Pessoal de Nível Superior-Brasil (CAPES)-Finance Code 1561551.

\section{Conflicts of Interest}

The authors declare no conflicts of interest regarding the publication of this paper.

\section{References}

[1] Pasupuleti, V.R., Prasad, T.N.V.K.V., Sheikh, R.A., Balam, S.K., Narasimhulu, G., 
Reddy, C.S., Rahman, I.A. and Gan, S.H. (2013) Biogenic Silver Nanoparticles Using Rhinacanthus nasutus Leaf Extract: Synthesis, Spectral Analysis, and Antimicrobial Studies. International Journal of Nanomedicine, 8, 3355-3364. https://doi.org/10.2147/IJN.S49000

[2] Raj, D.R., Prasanth, S., Vineeshkumar, T.V. and Sudarsanakumar, C. (2016) Surface Plasmon Resonance Based Fiber Optic Dopamine Sensor Using Green Synthesized Silver Nanoparticles. Sensors and Actuators B: Chemical, 224, 600-606. https://doi.org/10.1016/j.snb.2015.10.106

[3] Eising, R., Signori, A.M., Fort, S. and Domingos, J.B. (2011) Development of Catalytically Active Silver Colloid Nanoparticles Stabilized by Dextran. Langmuir, 27, 11860-11866. https://doi.org/10.1021/la2029164

[4] Vilas, V., Philip, D. and Mathew, J. (2014) Catalytically and Biologically Active Silver Nanoparticles Synthesized Using Essential Oil. Spectrochimica Acta Part A: Molecular and Biomolecular Spectroscopy, 132, 743-750.

https://doi.org/10.1016/j.saa.2014.05.046

[5] Hongfang, G., Hui, Y. and Chuang, W. (2017) Controllable Preparation and Mechanism of Nano-Silver Mediated by the Microemulsion System of the Clove Oil. Results in Physics, 7, 3130-3136. https://doi.org/10.1016/j.rinp.2017.08.032

[6] Raja, S., Ramesh, V. and Thivaharan, V. (2017) Green Biosynthesis of Silver Nanoparticles Using Calliandra haematocephala Leaf Extract, Their Antibacterial Activity and Hydrogen Peroxide Sensing Capability. Arabian Journal of Chemistry, 10, 253-261. https://doi.org/10.1016/j.arabjc.2015.06.023

[7] Keshari, A.K., Srivastava, R., Singh, P., Yadav, V.B. and Nath, G. (2018) Antioxidant and Antibacterial Activity of Silver Nanoparticles Synthesized by Cestrum nocturnum. Journal of Ayurveda and Integrative Medicine, in Press.

[8] Alizadeh, S., Ghoshal, S. and Comeau, Y. (2019) Fate and Inhibitory Effect of Silver Nanoparticles in High Rate Moving Bed Biofilm Reactors. Science of the Total Environment, 647, 1199-1210. https://doi.org/10.1016/j.scitotenv.2018.08.073

[9] Radünz, M., da Trindade, M.L.M., Camargo, T.M., Radünz, A.L., Borges, C.D., Gandra, E.A. and Helbig, E. (2018) Antimicrobial and Antioxidant Activity of Unencapsulated and Encapsulated Clove (Syzygium aromaticum, L.) Essential Oil. Food Chemistry, 276, 180-186. https://doi.org/10.1016/j.foodchem.2018.09.173

[10] Vijayaraghavan, K., Nalini, S.P.K., Prakash, N.U. and Madhankumar, D. (2012) Biomimetic Synthesis of Silver Nanoparticles by Aqueous Extract of Syzygium aromaticum. Materials Letters, 75, 33-35. https://doi.org/10.1016/j.matlet.2012.01.083

[11] Mashwani, Z., Khan, M.A., Khan, T. and Nadhman, A. (2016) Applications of Plant Terpenoids in the Synthesis of Colloidal Silver Nanoparticles. Advances in Colloid and Interface Science, 234, 132-141. https://doi.org/10.1016/j.cis.2016.04.008

[12] NCCLS (2012) Methods for Dilution Antimicrobial Susceptibility Tests for Bacteria That Grow Aerobically. NCCLS Document M7-A9. Approved Standard. Seventh Edition, Which Describes Standard Broth Dilution (Macrodilution and Microdilution) and Agar Dilution Techniques for Measuring the in Vitro Susceptibility of Bacteria to Antimicrobial Agents.

[13] Nunes, M.R., de Souza, M.C.M., de Lima, V.A.P., da Rosa, C.G., Noronha, C.M., Maciel, M.V.O.B and Barreto, P.M. (2018) Antioxidant and Antimicrobial Methylcellulose Films Containing Lippia alba Extract and Silver Nanoparticles. Carbohydrate Polymers, 192, 37-43. https://doi.org/10.1016/j.carbpol.2018.03.014

[14] Sathishkumar, M., Sneha, K., Won, S.W., Cho, C.-W., Kim, S. and Yun, Y.-S. (2009) Cinnamon zeylanicum Bark Extract and Powder Mediated Green Synthesis of Na- 
no-Crystalline Silver Particles and Its Bactericidal Activity. Colloids and Surfaces B: Biointerfaces, 73, 332-338. https://doi.org/10.1016/j.colsurfb.2009.06.005

[15] Jadhav, K., Dhamecha, D., Bhattacharya, D. and Patil, M. (2016) Green and Ecofriendly Synthesis of Silver Nanoparticles: Characterization, Biocompatibility Studies and Gel Formulation for Treatment of Infections in Burns. Journal of Photochemistry \& Photobiology, B: Biology, 155, 109-115.

https://doi.org/10.1016/j.jphotobiol.2016.01.002

[16] Xu, L., Peng, J., Yan, M., Zhang, D. and Shen, A.Q. (2016) Droplet Synthesis of Silver Nanoparticles by a Microfluidic Device. Chemical Engineering and Processing, 102, 186-193. https://doi.org/10.1016/j.cep.2016.01.017

[17] Song, Y., Li, R., Sun, Q. and Jin, P. (2011) Controlled growth of Cu Nanoparticles by a Tubular Microfluidic Reactor. Chemical Engineering Journal, 168, 477-484. https://doi.org/10.1016/j.cej.2011.01.059

[18] Pal, S., Tak, Y.K. and Song, J.M. (2007) Does the Antibacterial Activity of Silver Nanoparticles Depend on the Shape of the Nanoparticle? A Study of the Gram-Negative Bacterium Escherichia coli. Applied and Environmental Microbiology, 73, 1712-1720. https://doi.org/10.1128/AEM.02218-06 